\title{
Strategy for SAR ADC with $87.5 \%$ area saving and $99.4 \%$ switching energy reduction over conventional approach
}

\author{
Yuhua Liang, Zhangming Zhu ${ }^{\text {a)}, ~ J i a n ~ L i u, ~ a n d ~ R u i x u e ~ D i n g ~}$ \\ School of Microelectronics, Xidian University, Xi'an 710071, P. R. China \\ a)zhangmingzhu@xidian.edu.cn
}

\begin{abstract}
A state-of-the-art energy-efficient switching scheme for successive approximation register (SAR) analogue-to-digital converter (ADC) is proposed in this letter. With this proposed switching scheme, the average switching energy can be reduced by $98.8 \%$ compared to the conventional approach, and $75 \%$ of the chip area can be saved. While combining with an integer-based split-capacitor structure, both the average switching energy and chip area can be approximately reduced further by half. Yet, nonlinearity analysis and Monte Carlo simulation indicate that perfect linearity is still maintained.
\end{abstract}

Keywords: energy-efficient, SAR, ADC, switching energy

Classification: Integrated circuits

\section{References}

[1] C. C. Liu, S.-J. Chang, G.-Y. Huang and Y.-Z. Lin: IEEE J. Solid-State Circuits 45 (2010) 731. DOI:10.1109/JSSC.2010.2042254

[2] Y. Zhu, C.-H. Chan, U-F. Chio, S.-W. Sin, S.-P. U, R. P. Martins and F. Maloberti: IEEE J. Solid-State Circuits 45 (2010) 1111. DOI:10.1109/JSSC. 2010.2048498

[3] V. Hariprasath, J. Guerber, S.-H. Lee and U.-K. Moon: Electron. Lett. 46 (2010) 620. DOI:10.1049/el.2010.0706

[4] C. Yuan and Y. Lam: Electron. Lett. 48 (2012) 482. DOI:10.1049/el.2011.4001

[5] Z. Zhu, Y. Xiao and X. Song: Electron. Lett. 49 (2013) 327. DOI:10.1049/el. 2012.3332

[6] H. Song and M. Lee: IEICE Electron. Express 11 (2014) 20140345. DOI:10. 1587/elex.11.20140345

[7] L. Deng, C. Yang, M. Zhao, Y. Liu and X. Wu: New Circuits and Systems conference (NEWCAS) (2013) 1. DOI:10.1109/NEWCAS.2013.6573564

\section{Introduction}

SAR ADC has been the optimal choice to satisfy the requirement of low power 
included in SAR ADC, capacitive DACs contribute the bulk of total power. During the past several years, great efforts have been made to develop energy-efficient switching schemes for SAR ADCs to reduce the switching energy [1, 2, 3, 4, 5, 6]. Compared to the conventional switching scheme, the set-and-down scheme of [1] and $\mathrm{Vcm}$-based scheme of [2] realize a reduction of $81.2 \%$ and $87.5 \%$ separately in switching power. The MCS scheme of [3] and tri-level scheme of [4] can reach a reduction of $93.7 \%, 96.9 \%$, respectively. The Vcm-based monotonic scheme of [5], asymmetric monotonic switching scheme of [6] and the mixed switching scheme [7] can reach a reduction of $97.7 \%, 98.5 \%$ and $87.5 \%$, separately. However, with this proposed switching scheme, the switching energy and the chip area required can be reduced by $98.8 \%$ and $75 \%$ separately. While combined with the integerbased architecture, the switching energy and the chip area can be reduced further by half.

\section{Principle of energy saving for the capacitor array}

\subsection{Proposed switching scheme for SAR ADC}

Three effective solutions to improve the energy efficiency for SAR ADC is to optimize the switching scheme, to decrease the total number of capacitors utilized, and to minish the capacitance of the unit-capacitor $\mathrm{C}_{\mathrm{u}}$.

The proposed switching scheme for 4-bit SAR ADC is illustrated in Fig. 1. Adopting top-plated sampling technology, the first comparison is performed directly after sampling, and the switching energy is zero. The bottom-plates of capacitors are initially loaded with the sequence of $[\mathrm{Vcm}, 0, \cdots, 0]$, where $\mathrm{Vcm}$, representing the common-mode voltage, is half of Vref. Once the MSB is obtained, if Vip > Vin, all the capacitors at the positive side remain unchanged while at the negative side, the bottom-plate of the MSB capacitor is switched from $\mathrm{Vcm}$ to Vref, and the bottom-plates of all the LSBs are all switched from 0 to $\mathrm{Vcm}$. This operation would level the voltage at the negative side up by $1 / 2$ Vref, but consumes no energy. The case of Vip $<$ Vin is dealt with in the similar way. After the second comparison circle, if Vip > Vin, the voltage on the bottom-plate of the MSB capacitor at the positive side is decreased by $1 / 2 \mathrm{Vref}$, while all the other capacitors remain unchanged. This operation would lower the voltage at the positive side by $1 / 4 \mathrm{Vref}$, and also consumes no energy. The case of Vip $<$ Vin is also dealt with in the similar way.

Based on the above analysis, the conclusion that no energy is consumed during the first three comparison circles can be drawn. Since the bulk of the switching energy is consumed during the first several comparison circles, the proposed switching scheme would be more energy efficient than the other existing schemes. The average switching energy for 10-bit SAR with the proposed switching scheme proves to be $15.8 \mathrm{C}_{\mathrm{Vref}}{ }^{2}$, reduced by $98.8 \%$ compared to that with the conventional scheme.

\subsection{Proposed architecture of the capacitive DAC}

Switching the dummy capacitor only at one side of the capacitor array, either the positive side or the negative side, enables us to obtain the LSB bit. Together with 

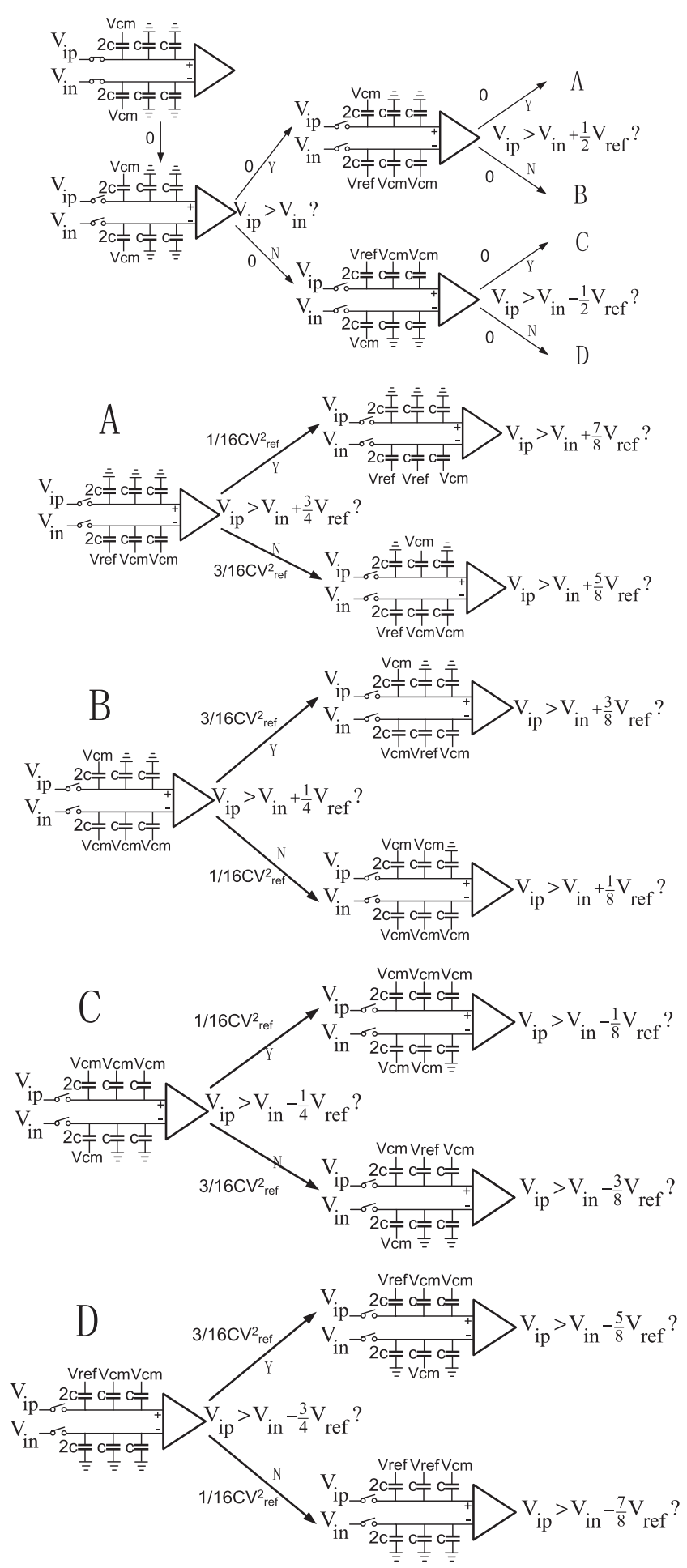

Fig. 1. Proposed switching scheme for 4-bit SAR ADC.

top-plated sampling technology, the number of the total capacitors achieves $4 \times$ reduction over the conventional approach. In this letter, a 2-times integer-based split capacitor array [7] is employed to improve the energy efficiency and save chip area further. Fig. 2a shows the capacitor array utilized in Fig. 1, while Fig. 2b shows the proposed capacitor array. Consequently, for N-bit SAR ADC, the number of capacitors Num can be reduced to:

$$
N u m=2 \times\left(2^{N-3}+3\right) C_{u}
$$


It is approximately $2 \times$ reduction over the schemes proposed in $[3,4,5,6]$, that is, $8 \times$ reduction over the conventional approach.

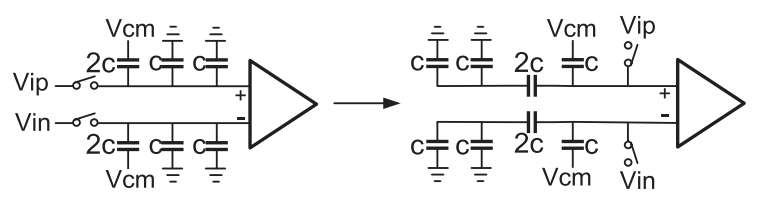

(a)

(b)

Fig. 2. Proposed integer-based split capacitor array for 4-bit SAR ADC

\subsection{Switching energy and nonlinearity analyses}

Combining the proposed switching scheme and the integer-based split capacitor array, a more energy-efficient and area-saving strategy for SAR ADC is realized. For the case of N-bit resolution, the average switching energy can be fit by the following formula.

$$
E_{\text {aver }}=\frac{1}{2} \sum_{i=1}^{N-2}\left(2^{N-i-6}\right) C V_{r e f}^{2}, N \geq 4
$$

Static performance of SAR ADC depends on both the adopted switching scheme and the employed architecture of capacitor array. Due to the MSB is mismatch independent, the worst case DNL with the proposed switching scheme occurs at $1 / 4 \mathrm{VFS}$ and $3 / 4 \mathrm{VFS}$. Together with the split capacitor, the voltages across $2 \times\left(2^{\mathrm{N}-3}+2\right) \mathrm{C}_{\mathrm{u}}$-elements are changed at this time. With fully differential structure, LSB turns out to be Vref $/ 2^{\mathrm{N}-1}$. Assuming the unit capacitor satisfies a nominal value of $\mathrm{C}_{\mathrm{u}}$ and a standard deviation of $\sigma\left(\mathrm{C}_{\mathrm{u}}\right)$, the standard deviation of the maximum DNL can be deduced as follows:

$$
\sigma_{D N L, M A X} \approx \frac{\sqrt{2^{N / 2}+18 \times 2^{2(N-6)}} \times \sigma(C u)}{C u}
$$

The former part under the root in formula (3) is generated by the switched capacitor while the latter part is generated by the split capacitor. So mismatch of the split capacitor would be a big restriction for high resolution. Fortunately, resolution for SAR ADC under low-power application conditions is usually no higher than 10-bit. In this case, the DNL performance depicted in (3) would not be worse than that with the conventional switching scheme on condition of the same resolution.

\section{Simulation results}

As Fig. 3 shown, on the condition of the same unit capacitor $\mathrm{C}_{\mathrm{u}}$, behavior simulation for 10-bit differential SAR ADC is performed to make the comparison of the switching energy between this proposed switching scheme and the other existing schemes. It can be seen that the energy efficiency with the proposed switching scheme proves to be optimal.

Some specific indicators for the schemes are listed in Table I. The average switching energy with the latest published scheme, the asymmetric monotonic switching scheme, is $19.84 \mathrm{CV}_{\text {ref }}{ }^{2}$, while with the proposed scheme, it is only 


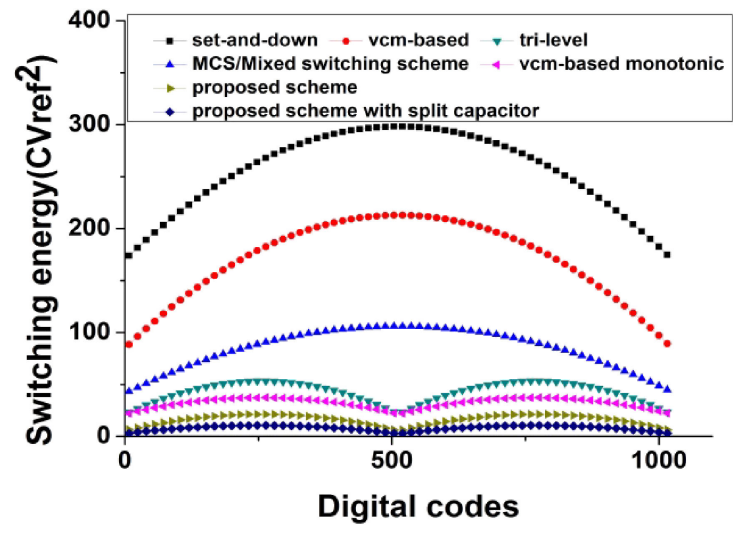

Fig. 3. Switching energy comparison for 10-bit SAR during transitions

Table I. Comparison of different switching schemes for 10-bit SAR.

\begin{tabular}{|c|c|c|c|}
\hline Switching scheme & $\begin{array}{c}\text { Average } \\
\text { switching energy }\end{array}$ & $\begin{array}{c}\text { Energy } \\
\text { saving }\end{array}$ & $\begin{array}{c}\text { Area } \\
\text { saving }\end{array}$ \\
\hline Conventional & $1363.33 \mathrm{CV}_{\text {ref }}^{2}$ & Reference & Reference \\
\hline Set-and-down [1] & $255.5 \mathrm{CV}_{\text {ref }}^{2}$ & $81.2 \%$ & $50 \%$ \\
\hline Vcm-based [2] & $170.2 \mathrm{CV}_{\text {ref }^{2}}$ & $87.5 \%$ & $50 \%$ \\
\hline MCS [3] & $85.1 \mathrm{CV}_{\text {ref }}^{2}$ & $93.7 \%$ & $75 \%$ \\
\hline Tri-level [4] & $42.4 \mathrm{CV}_{\text {ref }}^{2}$ & $96.9 \%$ & $75 \%$ \\
\hline asymmetric monotonic switching [6] & $31.9 \mathrm{CV}_{\text {ref }}^{2}$ & $97.7 \%$ & $75 \%$ \\
\hline Mixed switching scheme [7] & $19.84 \mathrm{CV}_{\text {ref }}^{2}$ & $98.5 \%$ & $75 \%$ \\
\hline Proposed switching scheme & $170.2 \mathrm{CV}_{\text {ref }^{2}}^{2}$ & $87.5 \%$ & $87.4 \%$ \\
\hline Proposed scheme with split-capacitor & $7.8 \mathrm{CV}_{\text {ref }^{2}}^{2}$ & $98.8 \%$ & $75 \%$ \\
\hline \multicolumn{2}{|r|}{} & $99.4 \%$ & $87.4 \%$ \\
\hline
\end{tabular}

$15.8 \mathrm{CV}_{\text {ref }}{ }^{2}$. Therefore, a reduction of $98.8 \%$ in the switching energy is realized compared with the conventional scheme and it would be state of the art.

Together with the integer-based-split architecture, the proposed switching scheme can reduce the switching energy by $99.4 \%$ and save $87.5 \%$ the chip area 500-runs Monte-Carlo simulation on nonlinearity performance for 10-bit SAR $\mathrm{ADC}$ is performed in MATLAB, and a comparison is made between the conventional approach and the proposed strategy described in this letter. Let the mismatch of unit capacitor satisfy $\sigma=5 \%$, simulation results are shown in Fig. 4. Fig. 4a corresponds to the conventional approach, while Fig. $4 \mathrm{~b}$ to the proposed strategy. It can be seen that the standard deviations of DNL and INL, with the conventional approach and the proposed strategy, are approximately equal. The root-meansquare (RMS) DNL and root-mean-square INL for each digital code are also simulated as Fig. 4 shown. Obviously, the maximum root-mean-square DNLs and the maximum root-mean-square INLs remain nearly equal.

As previously referred, resolution for low-power applications would be no higher than 10-bit. With the resolution down, it can be predicted by (3) that performance with the proposed strategy would be superior to that with the conventional approach. 

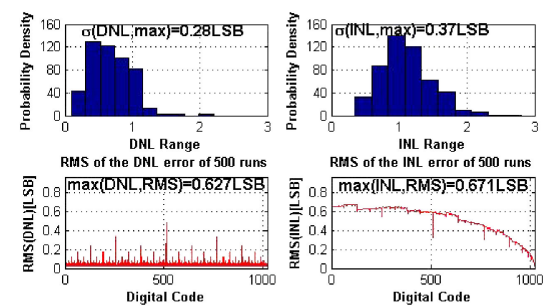

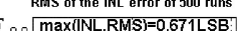

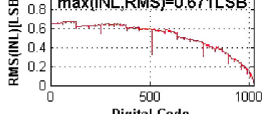

(a)

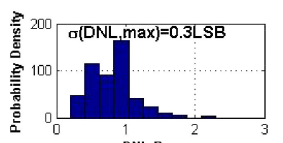

DNL Range

RMS of the DNL error of 500 runs

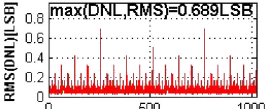

500
Digital Cade

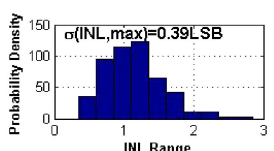

INL Range
RMS of the INL error of 500 runs 垔 0.8 max(INL,RMS $)=0.731$ LSB

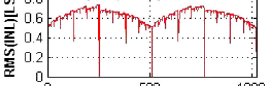

(b)

Fig. 4. Comparison of maximum DNL and INL for 10-bit SAR ADC

\section{Conclusions}

A high energy-efficiency switching scheme together with the integer-based split capacitor for SAR ADC is proposed in this letter. Compared with other existing schemes published so far, the proposed scheme achieves a reduction of $99.4 \%$ in switching energy and saves $87.4 \%$ of the chip area, yet still possesses of good linearity performance.

\section{Acknowledgments}

This work was supported by the National Natural Science Foundation of China (61234002, 61322405, 61306044, 61376033), the National High-tech Program of China (2013AA014103). 